\title{
Immunolocalization of MMP9 and MMP2 in osteolytic metastasis originating from MDA-MB-231 human breast cancer cells
}

\author{
BO LIU ${ }^{1}$, JIAN CUI $^{1}$, JING SUN $^{1}$, JUAN LI $^{1}$, XIUCHUN HAN $^{1}$, JIE GUO $^{1}$, \\ $\mathrm{MIN} \mathrm{YI}^{2}$, NORIO AMIZUKA ${ }^{3}, \mathrm{XIN} \mathrm{XU}^{1}$ and MINQI LI ${ }^{1}$ \\ ${ }^{1}$ Department of Bone Metabolism, School of Stomatology, Shandong University, \\ Shandong Provincial Key Laboratory of Oral Tissue Regeneration, Jinan, Shandong 250012, P.R. China; \\ ${ }^{2}$ Department of Advanced Medicine, Graduate School of Medicine, Hokkaido University, Sapporo, Hokkaidō 060-8638; \\ ${ }^{3}$ Department of Developmental Biology of Hard Tissue, Graduate School of Dental Medicine, \\ Hokkaido University, Sapporo, Hokkaidō 060-8586, Japan
}

Received July 13, 2015; Accepted May 16, 2016

DOI: $10.3892 / \mathrm{mmr} .2016 .5374$

\begin{abstract}
The aim of the present study was to investigate the expression of matrix metalloproteinase (MMP)9 and MMP2, and their potential roles in bone metastasis nests using a well-standardized model of breast cancer bone metastasis in nude mice. BALB/c nu/nu mice (5-week-old; $\mathrm{n}=10$ ) were subjected to intracardiac injection of MDA-MB-231 human breast cancer cells. After 4 weeks, the mice exhibiting radiolucent lesions in tibiae were sacrificed, and the tibiae were removed for histochemical analysis. The gene expression of MMP2 and MMP9 in the tumor cells, metaphysis and diaphysis of normal $\mathrm{BALB} / \mathrm{c} \mathrm{nu} / \mathrm{nu}$ mice were determined using reverse transcription-polymerase chain reaction analysis. The metastatic tumor tissue occupied almost the entire bone marrow cavity. Numerous tartrate-resistant acid phosphatase-positive osteoclasts were found in the metastasized lesions. The invaded tumor cells positive for mammaglobin 1 exhibited different proliferation activities and apoptosis between the metaphysis and diaphysis. Proliferating cell nuclear antigen was expressed at high levels in the metaphyseal area, whereas TdT-mediated dUTP nick-end labeling (TUNEL)-positive cells were more evident in the diaphysis area. Of note, MMP9 was expressed predominantly in the proliferating cell nuclear antigen-positive area, whereas the expression of MMP2 was observed predominantly in the diaphysis, which had more TUNEL-positive cells. Taken together, the results suggested that MMP9 and MMP2 may have their own importance in extracellular matrix degradation and trabecular bone damage
\end{abstract}

Correspondence to: Professor Minqi Li, Department of Bone Metabolism, School of Stomatology, Shandong University, Shandong Provincial Key Laboratory of Oral Tissue Regeneration, 44-1 Wenhua West Road, Jinan, Shandong 250012, P.R. China E-mail: liminqi@sdu.edu.cn

Key words: matrix metalloproteinase 2, matrix metalloproteinase 9, proliferation, apoptosis, bone metastasis in different zones of bone metastasis, including the metaphysis and diaphysis.

\section{Introduction}

Breast cancer is one of the types of cancer, which readily metastasizes to bone. Coleman (1) reported that between 65 and $75 \%$ of patients with advanced breast cancer develop bone metastases. Bone metastases are usually accompanied by pain, pathological fractures, nerve compression syndromes and hypercalcemia (2). Histomorphological analyses of bone metastases have revealed two types of lesions, osteolytic and osteogenetic. In bone metastasis of breast cancer, $80 \%$ of stage IV cases are found to be osteolytic and are accompanied by increased osteoclast activity (3). The process of osteoclastic bone resorption leads to the release of several cytokines, including transforming growth factor- $\beta$ (TGF- $\beta$ ) and insulin-like growth factors, which stimulate the proliferation and invasion of tumor cells, thus promoting a 'vicious cycle' of tumor metastasis and bone destruction (4).

In our previous studies, it was found that matrix metalloproteinases (MMPs) are important in the development and expansion of tumor cells in bone metastasis and skeletal osteolysis $(5,6)$. The degradation of extracellular matrix (ECM) by MMPs facilitates tumor cell invasion and proliferation in the metastatic environment (7-9). Among all MMP members, MMP1, 2, 3, 9 and 13 have been reported to correlate with tumor metastasis $(10,11)$. Lee et al demonstrated that the inhibition of MMP2 and MMP9 undermines the capability of bone degradation by tumor metastasis $(12,13)$. MMP2 is secreted predominantly by fibroblasts and osteoblasts $(14,15)$, and is involved in the activation of MMP13 (16) and degradation of the basement membrane (17). MMP9 is produced principally by osteoclasts (15) and cells of the immune system, including macrophages and neutrophils, which have been reported to be important for tumor growth $(10,18)$. MMP2 and MMP9 are able to cleave collagen type I, IV and V, and are important in the degradation of bone matrix (19).

Although the majority of the studies have focussed on host-derived MMPs, there have been few reports on the 
interrelation between MMPs and metastatic tumor cells. Therefore, the present study investigated the expression of MMPs in osteolytic bone metastasis nests originating from human breast cancer cells.

\section{Materials and methods}

Cell culture. Human MDA-MB-231 breast cancer cells were supplied by Professor Xiangzhi Li (Shandong University, Jinan, China). These cells were grown in RPMI 1640 media supplemented with $10 \%$ fetal bovine serum, $2 \mathrm{mM}$ glutamine, $1 \mathrm{mM}$ sodium pyruvate, $0.02 \mathrm{mM}$ non-essential amino acids and $1 \%$ streptomycin/penicillin at $37^{\circ} \mathrm{C}$ in a $5 \% \mathrm{CO}_{2}$ environment. All cells were cultured in $25 \mathrm{~cm}^{2}$ cell-culture flasks to $70-80 \%$ confluence.

Animal model of breast cancer bone metastasis and tissue preparation. All animal experiments were performed under the Guidelines for Animal Experimentation of Shandong University. The animal model of human breast cancer bone metastasis was established through intracardiac injection of the MDA-MB-231 human breast cancer cells into 5-week-old BALB/c nu/nu female mice (Vital River Laboratory Animal Technology Co. Ltd., Beijing, China) under anesthesia. On the day of injection, the flask-cultured MDA-MB-231 cells were trypsinized, counted with a hemocytometer, and diluted to a concentration of $2 \times 10^{6}$ cells $/ \mathrm{ml}$ in ice-cold Hank's balanced salt solution. Following anesthetization of the mice with $8 \%$ chloral hydrate $(400 \mathrm{mg} / 100 \mathrm{~g}$ body weight), a $0.1 \mathrm{ml}$ dilution ( $2 \times 10^{5}$ cells) was injected intracardially into the left ventricle of each mouse $(n=10)$, using a $1 \mathrm{ml}$ syringe, similar to a previously published method $(5,6)$. The mice were housed in micro-isolator solid-bottomed, polycarbonate cages under standard laboratory conditions with a 12-h light/dark cycle and a constant temperature of $20^{\circ} \mathrm{C}$ and humidity of $48 \%$. All mice were maintained on a standard commercial diet with autoclaved water available ad libitum. At 4 weeks post-injection, upon confirmation of an visible bone metastasis in the tibiae through soft X-ray analysis, the mice were anesthetized with an intraperitoneal injection of $10 \%$ chloral hydrate ( $400 \mathrm{mg} / 100 \mathrm{~g}$ body weight) and fixed with $4 \%$ formaldehyde in $0.1 \mathrm{M}$ phosphate buffer ( $\mathrm{pH}$ 7.4) by transcardial perfusion, and then the tibiae were removed for histological processing. Briefly, the samples were decalcified with $10 \%$ EDTA-2Na solution for 3 weeks at $4^{\circ} \mathrm{C}$. The specimens were dehydrated using an ascending ethanol series and then embedded in paraffin using standard procedures. Serial sections of $5 \mu \mathrm{m}$ in thickness were prepared for histochemical analysis.

Histological examination. Hematoxylin and eosin (H\&E) staining was performed to investigate the morphology of tibia in both groups. Following dewaxing and hydration, the prepared sections were immersed in Erthlich's haematoxylin for $15 \mathrm{~min}$. The sections were then washed with distilled water and differentiated in $1 \% \mathrm{HCl}$ in $70 \%$ alcohol for $1 \mathrm{~min}$ and washed again for 2 mins. Following this, the sections were stained with $1 \%$ eosin for $10 \mathrm{~min}$ and washed with distilled water. Subsequently, all sections were dehydrated and mounted. The stained sections were observed and then digital images were obstained using a light microscope (Olympus BX-53; Olympus Corportation, Tokyo, Japan).

Immunohistochemical examinations for mammaglobin 1 $(M G B 1)$, proliferating cell nuclear antigen (PCNA), MMP2, MMP9 and MMP13. The dewaxed paraffin sections were treated with $0.3 \%$ hydrogen peroxide for $30 \mathrm{~min}$ at room temperature, and then pre-incubated with $1 \%$ bovine serum albumin in phosphate-buffered saline (BSA-PBS) for $20 \mathrm{~min}$ at room temperature to reduce nonspecific binding. Subsequently, the sections were incubated with the following primary antibodies in BSA-PBS at room temperature for $2 \mathrm{~h}$ : SCGB2A2/mammaglobin A polyclonal antibody (MGB1; Proteintech; Sanying Biotechnology, Wuhan, China; cat. no. 235-1-AP; 1:50), anti-PCNA (Ab-1) mouse monoclonal antibody (PC101; Epitomics; Abcam, Burlingame, CA, USA; cat. no. NA03; 1:50), mouse anti-human MMP2 monoclonal antibody (EMD Millipore, Billerica, MA, USA; cat. no. MAB3308; 1:50), mouse MMP9 antibody antigen affinifty-purified polyclonal goat IgG (EMD Millipore; cat. no. AF909; 1:50) and goat anti-MMP-13 polyclonal antibody (EMD Millipore; cat. no. AB8120; 1:50). Following rinsing with PBS, the sections were incubated with the following secondary antibodies for $1 \mathrm{~h}$ at room temperature: Polyclonal swine anti-rabbit immunoglobulin(Ig)/HRP from DakoCytomation, Denmark (cat. no. Nr.P 0399; 1:100), goat polyclonal anti-mouse $\operatorname{IgG}+\operatorname{IgM}+\operatorname{Ig} \mathrm{A}-\mathrm{H} \& \mathrm{~L}$ (HRP) from Abcam (cat. no. ab102448; 1:100), goat polyclonal anti-mouse IgG+IgM+IgA-H\&L (HRP) from Abcam (cat. no. ab102448; 1:100), peroxidase-conjugated AffiniPure anti-goat++IgG $(\mathrm{H}+\mathrm{L})$ from Jackson Immunoresearch Laboratories, Inc. (West Grove, PA, USA; cat. no. 93894; 1:100) and peroxidase-conjugated AffiniPure anti-goat++IgG $(\mathrm{H}+\mathrm{L})$ from Jackson Immunoresearch Laboratories, Inc. (cat. no. 93894; 1:100). The immune complexes were then visualized using 3,3'-diamino-benzidine tetrahydrochloride (Sigma-Aldrich, St. Louis, MO, USA) as the substrate. All stained sections were faintly counterstained with methyl green for assessment using light microscopy (BX53; Olympus Corporation, Tokyo, Japan). The immunostaining intensities (optical density; OD) for all sections, with the exception of PCNA, were analyzed using Image-Pro Plus 6.2 software (Media Cybernetics, Inc., Silver Spring, MD, USA). Areas exhibiting a positive reaction were manually selected using a colour cube-based colour separate module in Image-Pro Plus. At least six sections from each sample were analyzed. All values are presented as the mean \pm standard deviation. The differences in OD values between the metaphysis and diaphysis for each immunostaining, and differences in the OD values between MMP2 and MMP9 in the metaphysis and diaphysis were assessed using Student's $t$-test. Differences among the MMP2 immunointensity in metaphysis group, MMP9 immunointensity in the metaphysis group, MMP2 immunointensity in the diaphysis group and MMP9 immunointensity in the diaphysis group were analyzed using analysis of variance (ANOVA). Statistical analysis was performed using GraphPad Prism, version 5.0 (GraphPad Software, Inc., La Jolla, CA, USA). P<0.01 was considered to indicate a statistically significant difference.

Tartrate-resistant acid phosphatase (TRAP) staining for osteoclast localization. To evaluate the localization of osteoclasts, 

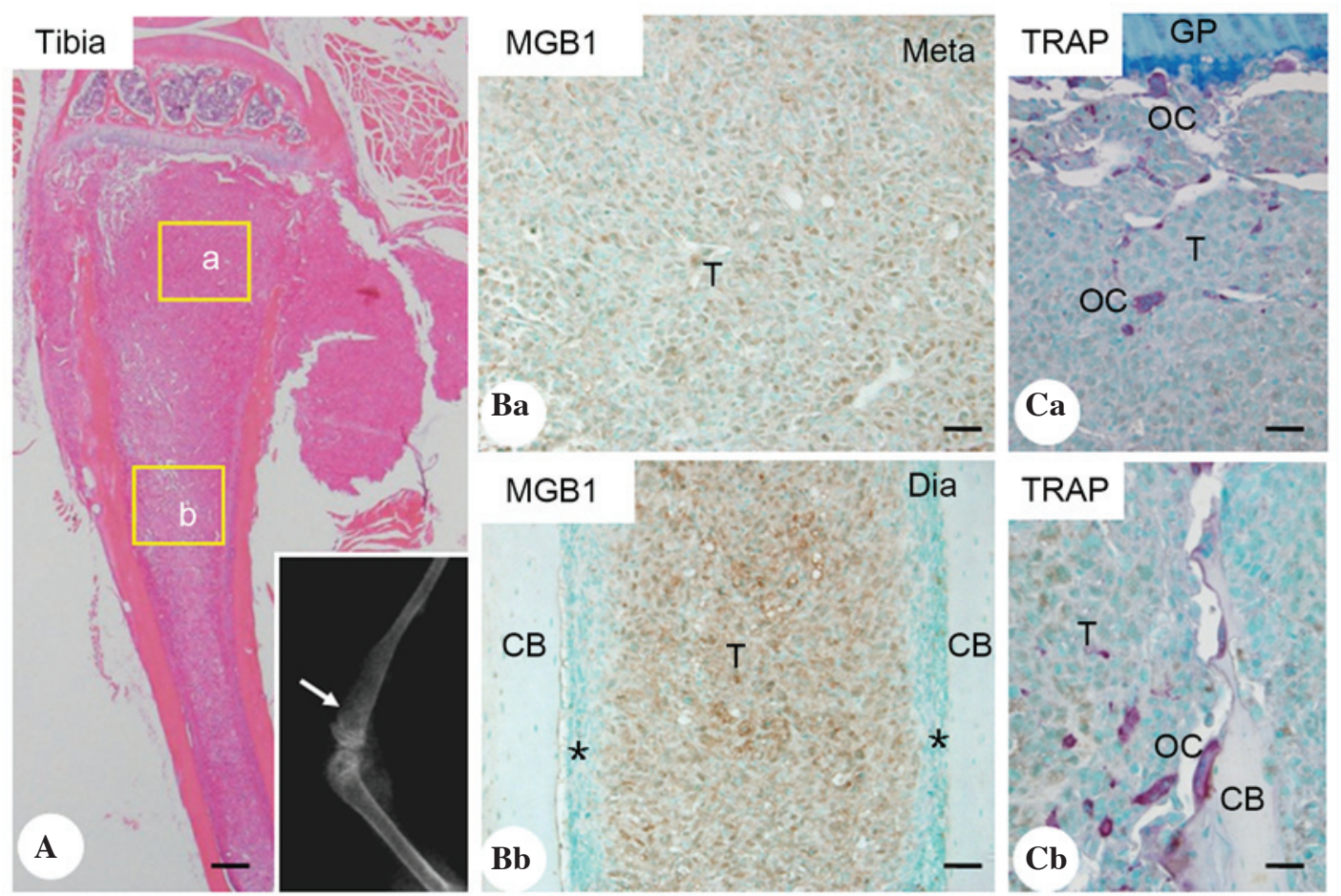

Figure 1. H\&E staining, soft X-ray examination and immunohistochemical analyses of MGB1 and TRAP staining. (A) H\&E staining and soft X-ray examination for breast cancer bone metastasis in tibiae. The metastatic tumor tissue occupied the majority of the spaces in the metaphysis and diaphysis. X-ray examination (lower right corner) revealed a radiolucent lesion in the tibia (white arrow). (Ba and Bb) Immunohistochemical analyses for MGB1 in the areas indicated by the yellow boxes in the (Aa) metaphysis and (Ab) diaphysis. Positive MGB1 staining (brown color) indicated metastatic breast cancer tissue. (Ca and b) Histochemical analysis of TRAP. Abundant TRAP-positive osteoclasts were found within the breast cancer metastasis nests and on the surface of the trabecular bone. Scale bars $=250 \mu \mathrm{m}$ for A; $50 \mu \mathrm{m}$ for B and C. T, tumor cells; CB, cortical bone, *, normal bone marrow tissue; H\&E, hematoxylin and eosin; MGB1, mammaglobin 1; TRAP, tartrate-resistant acid phosphatase.

TRAP staining was performed, as previously reported (20). In brief, the dewaxed paraffin sections were submerged in a mixture of $3.0 \mathrm{mg}$ naphthol AS-BI phosphate, $18 \mathrm{mg}$ red violet LB salt and $100 \mathrm{mM} \mathrm{L}(+)$ tartaric acid $(0.36 \mathrm{~g})$ diluted in $30 \mathrm{ml}$ of $0.1 \mathrm{M}$ sodium acetate buffer ( $\mathrm{pH} 5.0$ ) for $15 \mathrm{~min}$ at $37^{\circ} \mathrm{C}$. The sections were then faintly counterstained with methyl green for assessment using light microscopy (BX53; Olympus Corporation).

In situ detection of apoptosis in breast cancer bone metastasis. In order to identify the apoptotic status of the cells in the metastatic tissues, TdT-mediated dUTP nick-end labeling (TUNEL) analysis was performed using a TACS 2 TdT-blue label in situ apoptosis detection kit (cat. no. 4811-30-K; Trevigen, Inc., Gaithersburg, MD, USA). Briefly, the sections were placed in $1 \mathrm{X}$ PBS for $10 \mathrm{~min}$ at room temperature following rehydration in ethanol, and then covered with $50 \mu \mathrm{l}$ proteinase $\mathrm{K}$ solution and incubated for $15-30 \mathrm{~min}$ at $37^{\circ} \mathrm{C}$. Following washing twice in deionized water ( 2 min per wash), the sections were immersed in quenching solution for $5 \mathrm{~min}$ at room temperature. Following washing in $1 \mathrm{X}$ PBS for $1 \mathrm{~min}$ at room temperature, the sections were immersed in $1 \mathrm{X}$ TdT labeling buffer for $5 \mathrm{~min}$, following which they were covered with $50 \mu 1$ labeling reaction mix and incubated at $37^{\circ} \mathrm{C}$ for $1 \mathrm{~h} \mathrm{in}$ a humidity chamber. Subsequently, the sections were immersed in $1 \mathrm{X}$ TdT Stop buffer for $5 \mathrm{~min}$ at room temperature to terminate the labeling reaction. Following washing twice in 1X PBS for $5 \mathrm{~min}$ each at room temperature, the sections were covered in $50 \mu \mathrm{l}$ streptavidin-HRP solution and incubated for $10 \mathrm{~min}$ at $37^{\circ} \mathrm{C}$. Following washing twice in $1 \mathrm{X}$ PBS for 2 min each, the sections were immersed in TACS-Blue label solution for $4 \mathrm{~min}$, following which the samples were washed in several changes of deionized water for $2 \mathrm{~min}$ each. Finally, the samples were counterstained using nuclear fast red. The numbers of PCNAand TUNEL-positive cells were counted using Image pro Plus 6.2 software (Media Cybernetics, Inc., Silver Spring, MD, USA). The positively stained cells were manually selected. At least six sections from each sample were analyzed. All values are presented as the mean \pm standard deviation. Differences between the numbers of PCNA- and TUNEL-positive cells in the metaphysis/diaphysis were assessed using Student's $t$-test. Difference among the numbers of PCNA-positive cells in the metaphysis, TUNEL-positive cells in the metaphysis, PCNA-positive cells in the diaphysis and TUNEL-positive cell cells in the diaphysis, was analyzed using ANOVA. Statistical analysis was performed using GraphPad Prism, version 5.0 (GraphPad Software, Inc.). P $<0.01$ was considered to indicate a statistically significant difference.

RNA isolation and reverse transcription-polymerase chain reaction $(R T-P C R)$ analysis. The $\mathrm{BALB} / \mathrm{c}$ nu/nu mice were sacrificed by overdose of anesthesia. The tibiae were removed and separated into the metaphysis and diaphysis. For RT-PCR, total RNA were extracted from the MDA-MB-231 cells, metaphysis and diaphysis of the normal BALB/c nu/nu mice using TRIzol reagent (Invitrogen; Thermo Fisher Scientific, Inc., Waltham, MA, USA), according to the manufacturer's protocol. The first-strand complementary DNA was synthesized 
A
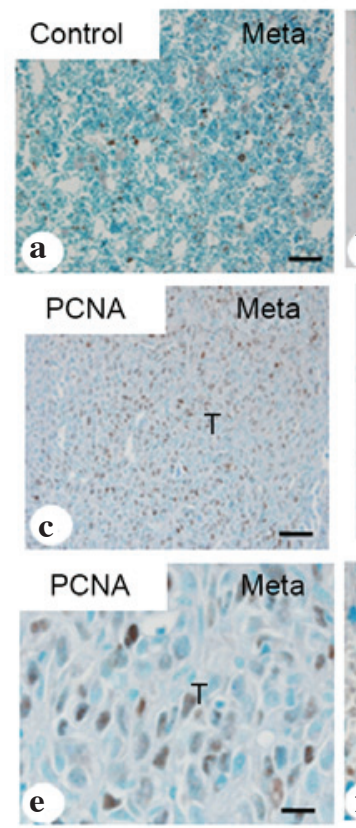
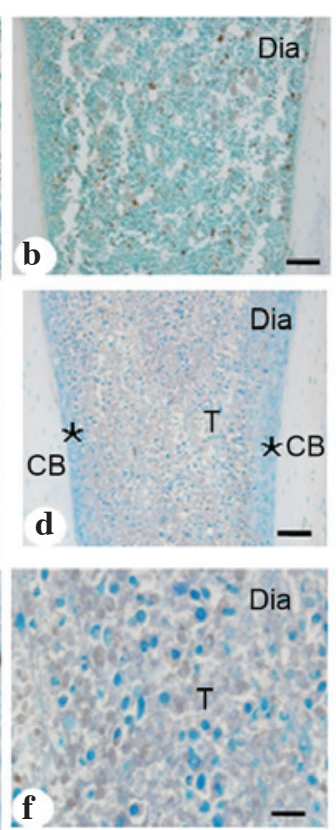

B
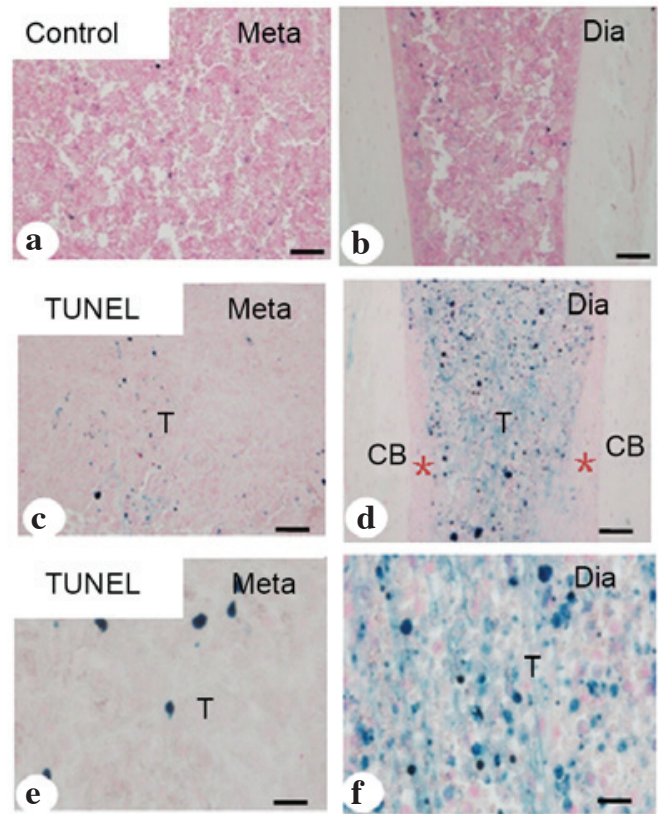

C

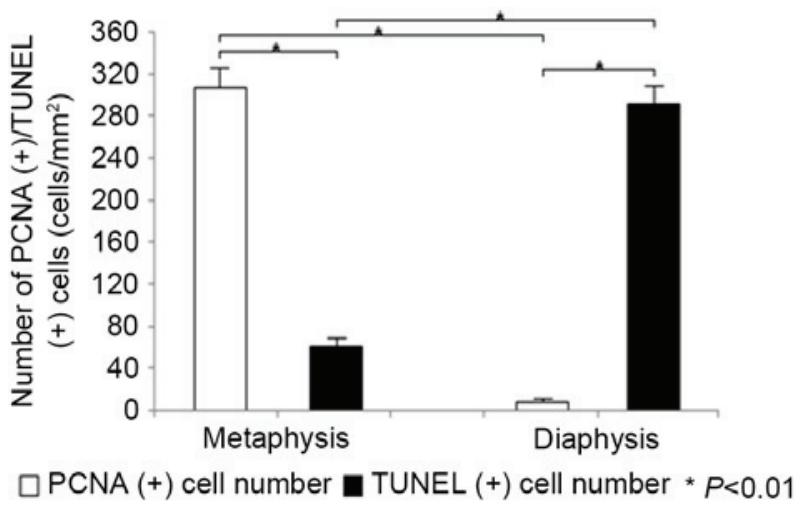

Figure 2. Immunohistochemical and statistical analyses of PCNA and TUNEL staining for apoptosis. (A) Immunohistochemical analysis of PCNA in the (a) metaphysis and (b) diaphysis in the normal bone marrow of the control group. Immunohistochemistry for PCNA in the (c) metaphyseal and (d) diaphyseal tumor metastases. More PCNA-positive tumor cells (brown color) were detected in the metaphyseal tumor tissue, compared with the diaphyseal tumor tissue. (e and f) Higher magnification of c and d, respectively. (B) TUNEL staining for apoptotic tumor cells in the (a) metaphysis and (b) diaphysis in the normal bone marrow tissue of the control group. TUNEL staining for apoptotic tumor cells in the (c) metaphyseal and (d) diaphyseal tumor metastases. More TUNEL-positive apoptotic tumor cells (blue color) were observed in the diaphyseal tumor tissue, compared with the metaphyseal tumor tissue. (e and f) High magnifications of $\mathrm{c}$ and d, respectively. (C) Statistical analyses of the numbers of PCNA/TUNEL-positive tumor cells in the metaphysis and diaphysis. ${ }^{*}<0.01$. Scale bar=50 $\mu \mathrm{m}$ in Aa-d and Ba-d; $25 \mu \mathrm{m}$ in Ae and f, and Be and f. TUNEL, TdT-mediated dUTP nick-end labeling; Meta, metaphysis; Dia, diaphysis; T, tumor cells; $\mathrm{CB}$, cortical bone; *, normal bone tissue.

using Superscript II reverse transcriptase (Invitrogen; Thermo Fisher Scientific, Inc.). The PCR analysis was performed using 2X Es Taq MasterMix (CWBio, Inc., Beijing, China) on a $100^{\mathrm{TM}}$ Thermal Cycler (Bio-Rad, Berkeley, CA, USA) and performed using the following primers: Human MMP-9 (Gene ID: 4318) sense 5'-GGG ACG CAG ACA TCG TCA TC-3' and antisense 5'-TCG TCA TCG TCG AAA TGG GC-3'), Human-MMP2 (Gene ID: 4313) sense 5'-GAT ACC CCT TTG ACG GTA AGGA-3' and antisense 5'-CCT TCT CCC A AG GTC CAT AGC-3', Mus-MMP9 (Gene ID: 17395) sense 5'-GCA GAG GCA TAC TTG TAC CG-3' and antisense 5'-TGA TGT TAT GAT GGT CCC ACT TG-3'; and Mus-MMP2 (Gene ID: 17390) sense 5'-ACC TGA ACA CTT TCT ATG GCTG-3' and antisense 5'-CTT CCG CAT GGT CTC GATG-3'. Human- $\beta$-actin (Gene ID: 60 ) sense 5'-CAT GTA CGT TGC TAT CCA GGC-3' and antisense 5'-CTC
CTT AAT GTC ACG CAC GAT-3' and Mus- $\beta$-actin (Gene ID: 11461) sense 5'-GGC TGT ATT CCC CTC CAT CG-3' and antisense 5'-CCA GTT GGT AAC AAT GCC ATGT-3' were used as internal controls (gene IDs from www.ncbi.nlm. nih.gov/gene/). The conditions for RT-PCR were similar to those previously described (21). The amplified PCR products were separated on $2 \%$ agarose gels and digitized using the SmartGel ${ }^{\mathrm{TM}}$ Image Analysis system (Sagecreation, Beijing, China).

\section{Results}

Development of breast cancer bone metastasis and TRAP staining. At 4 weeks post-intracardiac injection of MDA-MB-231 cells, 7/10 mice developed osteolytic lesions in the tibia, detected on soft X-ray examination (Fig. 1A; 
A
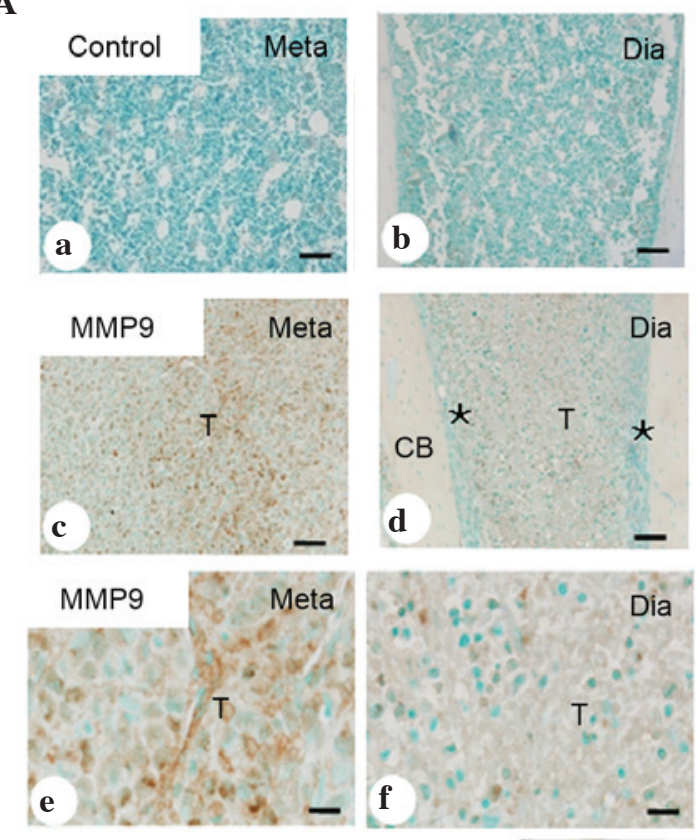

B
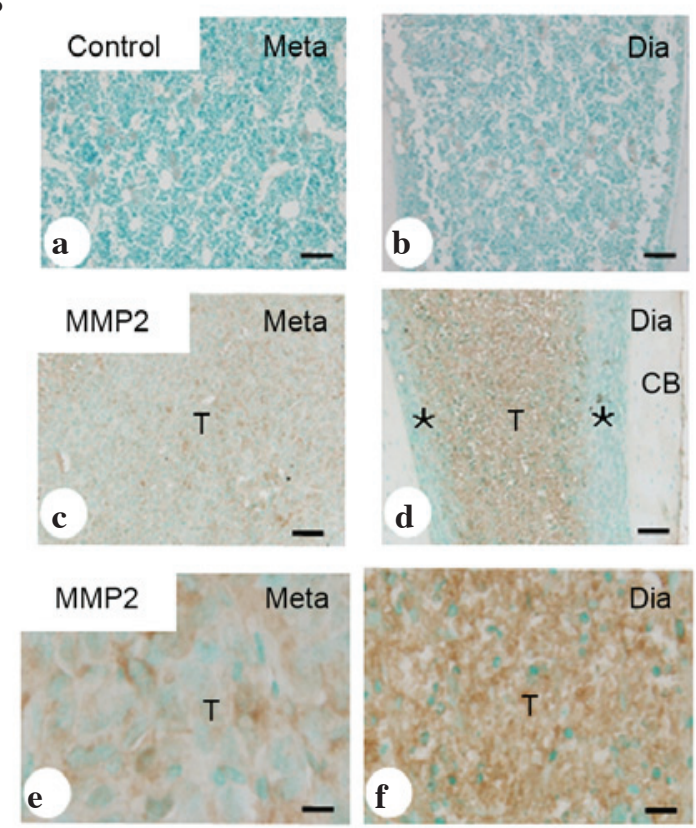

C

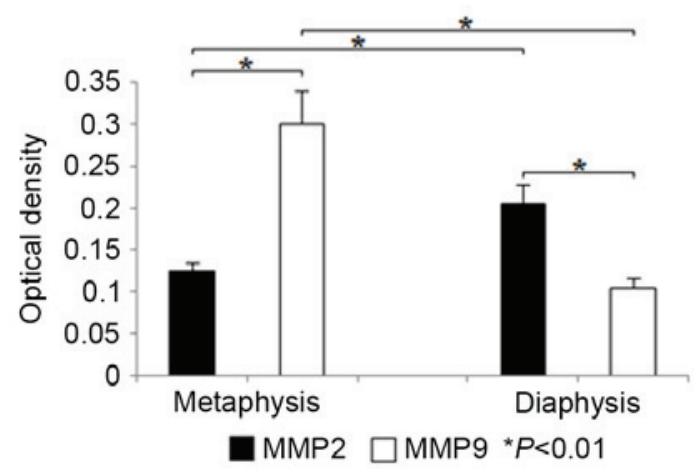

Figure 3. Immunohistochemical and statistical analyses of MMP9 and MMP2. (A) Immunohistochemical analysis of MMP9 in the (a) metaphysis and (b) diaphysis of normal bone marrow in the control group. Immunohistochemical analysis of MMP9 in tumor tissue of the (c) metaphysis and (d) diaphysis (brown color). The expression of MMP9 was significantly higher in the metaphysis, compared with the diaphysis. (e and f) High magnification of $\mathrm{c}$ and d, respectively. (B) Immunohistochemical analysis of MMP2 in the (a) metaphysis and (b) diaphysis of normal bone marrow in the control group. Immunohistochemical analysis of MMP2 in tumor tissue of the (c) metaphysis and (d) diaphysis (brown color). The expression of MMP2 was significantly higher in the diaphysis, compared with the metaphysis. (e and f) High magnification of c and d, respectively. (C) Statistical analyses of the immunostaining intensity of MMP9 and MMP2 in the metaphysis and diaphysis. ${ }^{*} \mathrm{P}<0.01$. Scale bars $=50 \mu \mathrm{m}$ in Aa-d and Ba-d; $25 \mu \mathrm{m}$ in Ae and $\mathrm{f}$, and Be and B. MMP, matrix metalloproteinase; Meta, metaphysis; Dia, diaphysis; T, tumor cells; CB, cortical bone; *, normal bone tissue.

white arrow). Breast cancer cells positive for MGB1, which are exclusively overexpressed in primary and metastatic human breast cancer (22), were abundant in the metastatic lesions of the metaphysis (Fig. 1B) and diaphysis (Fig. 1C). The TRAP staining showed that several TRAP-positive multinucleate cells were present within the breast cancer bone metastasis nests and on the surface of trabecular bone (Fig. 1D and E).

Immunolocalization of PCNA and in situ detection of apoptosis. PCNA-positive cells were observed in the metaphyseal area (Fig. 2Ab and c), but few were observed in the diaphyseal area (Fig. 2Ad and f). In the metaphyseal tumor nest, a few scattered TUNEL-positive apoptotic cells were observed (Fig. 2Bb and c). However, in the diaphyseal tumor nest, a higher number of TUNEL-positive tumor cells were present, compared with that in metaphyseal area (Fig. 2Be and f). ANOVA revealed that, in the metaphysis, the number of PCNA-positive cells was significantly higher, compared with the number of TUNEL-positive cells (307.78 \pm 27.04 , vs. $61.12 \pm 7.59$ cells $/ \mathrm{mm}^{2}$, respectively; $\mathrm{P}<0.01 ; \mathrm{n}=7$; Fig. $2 \mathrm{C}$ ). In the diaphysis, the number of TUNEL-positive cells was significantly higher, compared with the number of PCNA-positive cells $\left(291.96 \pm 20.78\right.$, vs. $8.04 \pm 1.09$ cells $/ \mathrm{mm}^{2}$, respectively; $\mathrm{P}<0.01$; $=7$; Fig. 2C).

Immunolocalization of MMP2, MMP9 and MMP13. In the metaphyseal area containing numerous PCNA-positive tumor cells, MMP9-immunopositivity was significantly more marked (Fig. 3Ab and c), whereas staining for MMP2 was faint (Fig. 3Bb and c). In contrast, the diaphyseal metastasis, containing more TUNEL-positive cells, showed weak expression of MMP9 (Fig. 3Ae and f), compared with MMP2 (Fig. 3Be and f). No significant differences were found in the immunolocalization and immunoreactivity of MMP13 between PCNA-positive and negative areas (data 


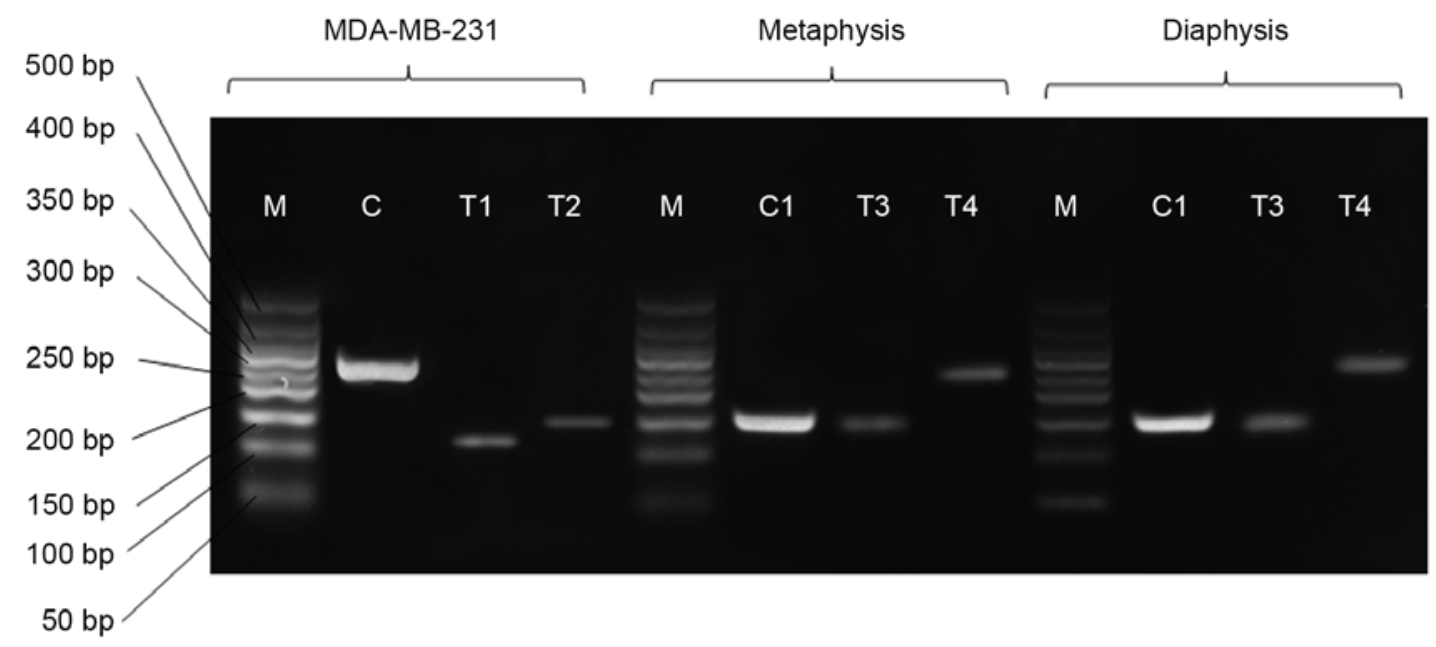

$\begin{array}{lccc}\text { M: DNA marker } 50 \mathrm{bp}-500 \mathrm{bp} & \text { C: human- } \beta \text {-actin } 250 \mathrm{bp} & \text { C1: Mus- } \beta \text {-actin } 154 \mathrm{bp} \\ \text { T1:Human-MMP2 } & 112 \mathrm{bp} & \text { T2:Human-MMP9 } & 139 \mathrm{bp} \\ \text { T3:Mus-MMP2 } & 140 \mathrm{bp} & \text { T4:Mus-MMP9 } & 229 \mathrm{bp}\end{array}$

Figure 4. Reverse transcription-polymerase chain reaction analysis of the mRNA expression of MMP2 and MMP9 in MDA-MB-231 cells, and the metaphysis and diaphysis of tibiae of BALB/c nu/nu mice. M, Tiangen DM:120227 DNA marker (50-500 bp) C, Human- $\beta$-actin (250 bp); C1, Mus- $\beta$-actin (154 bp); T1, target gene 1 (Human-MMP2) was positive in the MDA-MB-231 cells with a size of $112 \mathrm{bp}$ ). T2, target gene 2 (Human-MMP9) was positive in the MDA-MB-231 cells with a size of $139 \mathrm{bp}$ ). T3, target gene 3 (Mus-MMP2) was positive in the metaphysis of the tibiae of BALB/c nu/nu mice with a size of $140 \mathrm{bp}$ ). T4, target gene 4 (Mus-MMP9 was positive in the diaphysis of the tibiae of BALB/c nu/nu mice with a size of $229 \mathrm{bp}$. MMP, matrix metalloproteinase.

A

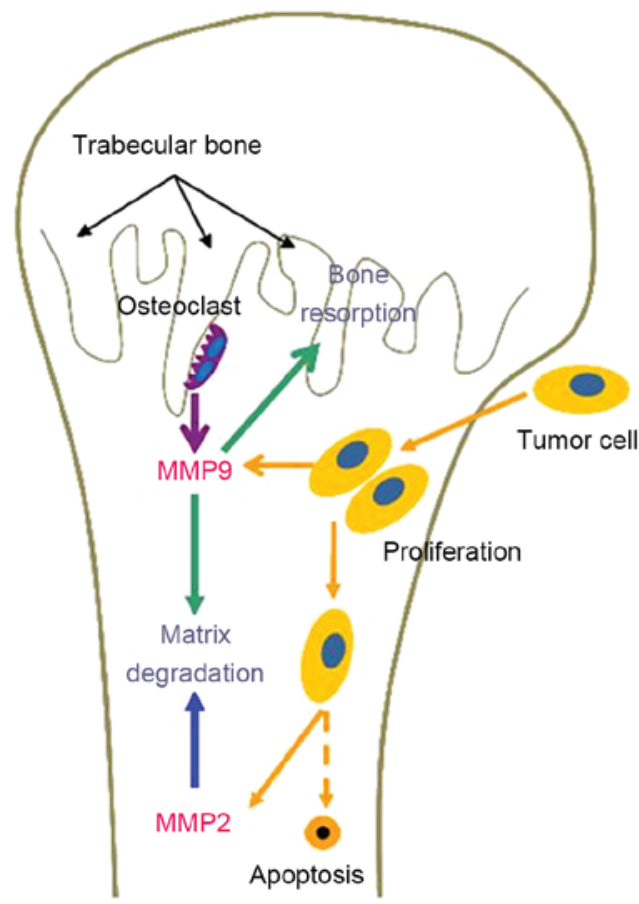

B

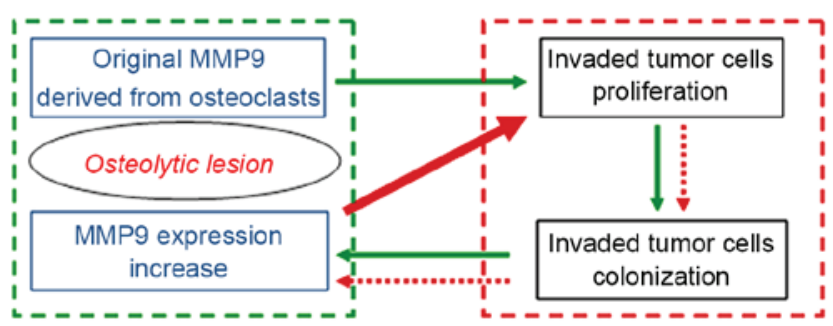

Figure 5. Schematic model showing the distribution and functions of MMP9 and MMP2 in tumor cell proliferation and colonization within the osseous microenvironment. (A and B) Following colonization in the metaphyseal microenvironment, the tumor cells exhibited a high level of MMP9 production, with dynamic proliferation activity. As tumor cells migrated to the diaphysis, the tumor derived expression of MMP9 decreased, whereas an increase in the expression of MMP2 was detected. However, this process was accompanied by a wave of tumor cell apoptosis. Tumor derived MMPs and host-derived MMPs further initiated bone matrix degradation. MMP, matrix metalloproteinase.

not shown). ANOVA revealed that, in the PCNA-positive metastatic area (metaphysis), the staining intensity of MMP2 was significantly weaker, compared with that of MMP9 $(0.126 \pm 0.007$, vs. $0.300 \pm 0.036$, respectively; $\mathrm{P}<0.01 ; \mathrm{n}=7$;
Fig. 3C). In the TUNEL-positive metastatic area (diaphysis), the staining intensity of MMP2 was significantly more marked, compared with that of MMP9 $(0.205 \pm 0.020$, vs. $0.103 \pm 0.009$; $\mathrm{P}<0.01$; Fig. 3 C). 
$R T-P C R$. The present study performed RT-PCR to investigate the source of MMP2 and MMP9. The results revealed that MMP2 and MMP9 mRNA were expressed in the MDA-MB-231 cells, metaphysis and diaphysis of the tibiae of the BALB/c nu/nu mice without tumor cell administration (Fig. 4).

\section{Discussion}

MMP9 and MMP2 belong to gelatinase, which is one of five groups of the MMP family, based on structure and substrate specificity (23). MMP9 and MMP2 are important in cancer invasion and metastasis by degrading the ECM and basement membrane (24). In the present study, the immunolocalization of MMP9 and MMP2 in osteolytic metastasis originating from human breast cancer cells were investigated. The results showed findings consistent with those of Ohshiba et al (15), that the expression levels of MMP9 and MMP2 were upregulated in bone metastasis nests. Notably, the present study found that MMP9 was overexpressed in the metaphysis with high expression levels of PCNA, whereas MMP2 was detected predominantly in the diaphysis with marked TUNEL-positive expression.

Metaphysis is the most common homing site for tumor cells due to its high level of vascularization. Once tumor cells home to metaphysis, they are stimulated to proliferate by MMP9 (25) and bone-derived growth factors, including TGF- $\beta$ (26), for their subsequent colonization in bone. Furthermore, Nutter et al demonstrated that the expression of MMP9 was increased on tumor cells colonization in bone (25). These findings were verified in the present study, which demonstrated that MMP9 was overexpressed in the metaphysis with a high level of PCNA-positive expression in the tumor cells (Fig. 5A). Tumor cells become the predominant source of MMP9 production with the extension of the bone metastasis nests, although MMP9 are predominantly derived from osteoclasts and vascular endothelial cells prior to tumor invasion. As shown in Fig. 5B, the 'vicious cycle', in which the original MMP9 derived from osteoclasts stimulates the proliferation of invaded tumor cells and subsequent colonization of tumor cells, accelerates the expression of MMP9 may provide a further explanation for tumor bone metastasis and offer a tumor prevention strategy. In addition, the increased MMP9 is involved in the recruitment of bone-resorbing osteoclasts, which leads to further osteolytic lesions $(27,28)$.

In the present study, the tumor cells appeared to expand towards the diaphysis following the initial invasion taking place in the metaphysis. Compared with the immunolocalization of MMP9, MMP2 was expressed at high levels in the diaphysis, which exhibits weak proliferation/increased apoptosis of tumor cells. Ni et al reported that the upregulation of MMP2 is important in breast cancer bone metastasis through the microRNA-106b/MMP2/extracellular signalregulated kinase pathway, which affects the balance of receptor activator of nuclear factor- $\kappa \mathrm{B}$ ligand and osteoprotegerin production (29). MMP2 is secreted predominantly by fibroblasts and osteoblasts $(14,15)$, however, the results of the present study showed negative expression in the fibroblasts and osteoblasts adjacent to the metastatic tumor cells in the diaphysis. Further investigation is required for understanding the intricate interactions among tumor cells and host bone marrow cells. In addition, based on existing data, it is difficult to explain why a higher number of TUNEL-positive cells were found in the diaphysis occupied by the invaded breast cancer cells. In view of a previous study, which demonstrated that breast cancer cells may induce osteoblast apoptosis (30), the present study hypothesized that the higher number of TUNEL-positive cells in the diaphysis may be composed predominantly of apoptotic stromal cells and fibroblasts induced by the invaded breast cancer cells. Although, certain apoptotic tumor cells may be contained due to decreased blood supply in the diaphysis.

In conclusion, the results of the present study showed that the invaded tumor cells exhibited different proliferation activity and apoptosis status between metaphysis and diaphysis. MMP9 was predominantly expressed in the PCNA-positive metaphysis, whereas MMP2 was predominantly expressed in the diaphysis, which contained more TUNEL-positive cells. As a consequence, it was suggested that MMP9 and MMP2 may have their own importance in ECM degradation and trabecular bone damage in different zones of bone metastasis. Further investigations are required to determine the exact mechanisms.

\section{Acknowledgements}

This study was partially supported by the National Nature Science Foundation of China (grant nos. 81271965, 81470719 and 81311140173) and the Specialized Research Fund for the Doctoral Program of Higher Education (grant no. 20120131110073) to Professor Minqi Li, and the Shandong Province Science and Technique Foundation, China (grant no. 2014GSF118093) to Dr Jie Guo .

\section{References}

1. Coleman RE: Metastatic bone disease: Clinical features, pathophysiology and treatment strategies. Cancer Treat Rev 27: 165-176, 2001.

2. Hamaoka T, Madewell JE, Podoloff DA, Hortobagyi GN and Ueno NT: Bone imaging in metastatic breast cancer. J Clin Oncol 22: 2942-2953, 2004.

3. Kozlow W and Guise TA: Breast cancer metastasis to bone: Mechanisms of osteolysis and implications for therapy. JMammary Gland Biol Neoplasia 10: 169-180, 2005.

4. Kingsley LA, Fournier PG, Chirgwin JM and Guise TA: Molecular biology of bone metastasis. Mol Cancer Ther 6: 2609-2617, 2007.

5. Li M, Amizuka N, Takeuchi K, Freitas PH, Kawano Y,Hoshino M, Oda K, Nozawa-Inoue K and Maeda T: Histochemical evidence of osteoclastic degradation of extracellular matrix in osteolytic metastasis originating from human lung small carcinoma (SBC-5) cells. Microsc Res Tech 69: 73-83, 2006.

6. Li M, Sasaki T, Ono K, de Freitas PH, Sobhan U, Kojima T, Shimomura J, Oda K and Amizuka N: Distribution of macrophages, osteoclasts and the B-lymphocyte lineage in osteolytic metastasis of mouse mammary carcinoma. Biomed Res 28: 127-137, 2007.

7. Jacob K, Webber M, Benayahu D and Kleinman HK: Osteonectin promotes prostate cancer cell migration and invasion: A possible mechanism for metastasis to bone. Cancer Res 59: 4453-4457, 1999.

8. Lynch CC and Matrisian LM: Matrix metalloproteinases in tumor-host cell communication. Differentiation 70: 561-573, 2002.

9. Nannuru KC, Futakuchi M, Varney ML, Vincent TM, Marcusson EG and Singh RK: Matrix metalloproteinase (MMP)-13 regulates mammary tumor-induced osteolysis by activating MMP9 and transforming growth factor-beta signaling at the tumor-bone interface. Cancer Res 70: 3494-3504, 2010. 
10. Egeblad M and Werb Z: New functions for the matrix metalloproteinases in cancer progression. Nat Rev Cancer 2: 161-174, 2002.

11. Scherer RL, Mclntyre JO and Matrisian LM: Imaging matrix metalloproteinases in cancer. Cancer Metastasis Rev 27: 679-690, 2008

12. Lee J, Weber M, Mejia S, Bone E, Watson P and Orr W: A matrix metalloproteinase inhibitor, batimastat, retards the development of osteolytic bone metastases by MDA-MB-231 human breast cancer cells in Balb C nu/nu mice. Eur J Cancer 37: 106-113, 2001.

13. Weber MH, Lee J and Orr FW: The effect of Neovastat (AE-941) on an experimental metastatic bone tumor model. Int J Oncol 20: 299-303, 2002.

14. Monteiro-Amado F, Castro-Silva II, Lima CJ, Soares FA, Kowalski LP and Granjeiro JM: Immunohistochemical evaluation of MMP-2, MMP-9 and CD31/microvascular density in squamous cell carcinomas of the floor of the mouth. Braz Dent J 24: 3-9, 2013.

15. Ohshiba T,Miyaura C,Inada Mand Ito A: Role of RANKL-induced osteoclast formation and MMP-dependent matrix degradation in bone destruction by breast cancer metastasis. Br J Cancer 88: 1318-1326, 2003.

16. Morrison C, Mancini S, Cipollone J, Kappelhoff R, Roskelley C and Overall C: Microarray and proteomic analysis of breast cancer cell and osteoblast co-cultures: Role of osteoblast matrix metalloproteinase (MMP)-13 in bone metastasis. J Biol Chem 286: 34271-34285, 2011.

17. Lafleur MA, Drew AF, de Sousa EL, Blick T, Bills M, Walker EC, Williams ED, Waltham M and Thompson EW: Upregulation of matrix metalloproteinases (MMPs) in breast cancer xenografts: A major induction of stromal MMP-13. Int J Cancer 114: 544-554, 2005 .

18. Huang S, Van Arsdall M, Tedjarati S, McCarty M, Wu W, Langley $\mathrm{R}$ and Fidler IJ: Contributions of stromal metalloproteinase-9 to angiogenesis and growth of human ovarian carcinoma in mice. J Natl Cancer Inst 94: 1134-1142, 2002.

19. Björklund M and Koivunen E: Gelatinase-mediated migration and invasion of cancer cells. Biochim Biophys Acta 1755: 37-69, 2005.

20. Li M, Hasegawa T, Hogo H, Tatsumi S, Liu Z, Guo Y, Sasaki M, Tabata C, Yamamoto T, Ikeda K and Amizuka N: Histological examination on osteoblastic activities in the alveolar bone of transgenic mice with induced ablation of osteocytes. Histol Histopathol 28: 327-335, 2013.
21. Tsunoda M and Sharma RP: Modulation of tumor necrosis factor alpha expression in mouse brain after exposure to aluminum in drinking water. Arch Toxicol 73: 419-426, 1999

22. Sasaki E, Tsunoda N, Hatanaka Y, Mori N, Iwata H and Yatabe Y: Breast-specific expression of MGB1/mammaglobin: An examination of 480 tumors from various organs and clinicopathological analysis of MGB1-positive breast cancers. Mod Pathol 20: 208-214, 2007

23. Slattery ML, John E, Torres-Mejia G, Stern M, Lundgreen A, Hines L, Giuliano A, Baumgartner K, Herrick J and Wolff RK: Matrix metalloproteinase genes are associated with breast cancer risk and survival: The Breast Cancer Health Disparities Study. PLoS One 8: e63165, 2013.

24. McCawley LJ and Matrisian LM: Matrix metalloproteinases: They're not just for matrix anymore! Curr Opin Cell Biol 13: 534-540, 2001

25. Nutter F, Holen I, Brown HK, Cross SS, Evans CA, Walker M, Coleman RE, Westbrook JA, Selby PJ, Brown JE and Ottewell PD: Different molecular profiles are associated with breast cancer cell homing compared with colonisation of bone: Evidence using a novel bone-seeking cell line. Endocr Relat Cancer 21: 327-341, 2014.

26. Yin JJ, Selander K, Chirgwin JM, Dallas M, Grubbs BG, Wieser R, Massagué J, Mundy GR and Guise TA: TGF-beta signaling blockade inhibits PTHrP secretion by breast cancer cells and bone metastases development. J Clin Invest 103: 197-206, 1999.

27. Woodward JK, Holen I, Coleman RE and Buttle DJ: The roles of proteolytic enzymes in the development of tumour-induced bone disease in breast and prostate cancer. Bone 41: 912-927, 2007.

28. Balbín M, Pendás AM, Uría JA, Jiménez MG, Freije JP and López-Otín C: Expression and regulation of collagenase-3 (MMP-13) in human malignant tumors. APMIS 107: 45-53, 1999.

29. Ni X, Xia T, Zhao Y, Zhou W, Wu N, Liu X, Ding Q, Zha X, Sha J and Wang S: Downregulation of miR-106b induced breast cancer cell invasion and motility in association with overexpression of matrix metalloproteinase 2. Cancer Sci 105: 18-25, 2014.

30. Mastro AM, Gay CV, Welch DR, Donahue HJ, Jewell J, Mercer R, DiGirolamo D, Chislock EM and Guttridge K: Breast cancer cells induce osteoblast apoptosis: A possible contributor to bone degradation. J Cell Biochem 91: 265-276, 2004. 